\title{
Examination of Color-Lighting Control System Using Colored Paper User Interface
}

\author{
Hiroto Aida ${ }^{1}$, Kento Matsui ${ }^{2}$, Soma Keisuke ${ }^{2}$, Hiroki Murakami ${ }^{2}$, Mistunori Miki ${ }^{1}$ \\ ${ }^{1}$ Department of Science and Engineering, Doshisha University, Japan \\ ${ }^{2}$ Graduate School of Science and Engineering, Doshisha University, Japan
}

\begin{abstract}
In recent year, Full-Color LED Lighting that can be changed to various color such as red, green, blue has been appeared with development of LED Lighting. By Color-Lighting control, users affected such as concentrating and relaxing. Therefore, Color-lighting control will spread to various place such as home, offices, stations. However color-lighting control affected some disturbance such as daylight, display when Full-Color LED controlled indoors. Also, information devices control get difficult with information technology develop. I propose Color-Lighting Control System using Colored Paper User Interface(CLC/CPUI). The purpose of CLC/CPUI is that anyone can intuitively control Full-Color LED Lighting. CLC/CPUI uses colored paper as user interface by sensing the paper. CLC/CPUI realizes lighting color that user demanded to do feedback control. I conduct accuracy verification experiment of CLC/CPUI.
\end{abstract}

\section{Introduction}

LED lighting has advanced drastically to draw attention with the advent of high-brightness blue LED. Compared with fluorescent lamps, LED lamps are characterized by less power consumption, long life, and easier downsizing. They are intended to be introduced into offices and homes. Full color LED lamps can produce light in various colors (colored light) such as red, green, and blue. It has been found that controlling a full color LED lamp to vary the brightness and color shade of light, in particular its color temperature, affects user's work efficiency and psychological relaxation [1, 2, 3, 4].

There is a problem, however, of a disturbance effect by light sources such as daylight, display, and task light in controlling the colored light of an individual LED lamp indoors. The impact of disturbance precludes providing the colored light environment demanded by a user appropriately due to a difference between the colored light environment supposed to be provided by the system and the one actually provided. Therefore, it is required to search a colored light demanded by the user by sensing the current colored light environment and performing feedback control.

With the development of information technology, information devices are loaded with a variety of functions, which complicates the operation of such devices. It is the same with full color LED lighting. While it is possible to realize a variety of colors and brightness by using a full color LED lamp, it is necessary to change multiple parameters to control it. As a result of such complication, a user who is unaccustomed to the operation of information devices finds it reluctant to use such devices. A method using a piece of paper is thus suggested as an intuitive way of operating a system. Chand et al. proposed Jadoo, a system using paper UI. We conducted a human subject experiment of Jadoo with users unaccustomed to the operation of information devices [5]. Based on this study, it is estimated that using a piece of paper instead of a keyboard and a mouse enables users unaccustomed to operating information devices to operate a device and leads them to master its use. In this way, using a piece of paper as UI is effective in making the use of information devices intuitive and letting everyone use them easily.

In this study, we thus constructed a Color-Lighting Control System using Colored Paper User Interface (CLC/CPUI), which realizes a colored light a user demands by using colored paper as UI sensed by a vision sensor for feedback control of a full color LED lamp, for the purpose of enabling everyone to control a full color LED lamp in an intuitive manner.

The organization of this paper is as follows. Section 2 gives some remarks on colored light and colors. Section 3 describes the Color-Lighting Control System using Colored Paper User Interface. Section 4 describes the verification experiment for CLC/CPUI. Section 5 gives the conclusion. 


\section{Colored Light and Color}

\subsection{Light Color and Object Color}

Colored light is light recognized to have color by human sensitivity. The color of colored light is called light color. Colored light is described by illuminance and chromaticity, which are used as indices in measurement in a colored light environment. Object color is color caused to appear by light reflected by the surface of an object or light permeating an object.

\subsection{Illuminance}

Illuminance is an indicator of brightness of an illuminated surface and represented by the unit of lux (lx). It has been found that appropriate illuminance differs by an individual's preference and the nature of work [6]. Japanese Industrial Standards (JIS) recommend working in different illuminance environments depending on the purpose of using a given space [7]. For instance, a high illuminance environment of $1, \mathrm{~nm}, .000 \mathrm{~lx}$ or greater is recommended if precise work such as drafting or assembling is required. Medium illuminance of about 750 $\mathrm{lx}$ is recommended for a general office, meeting room, classroom, etc.

The above criteria, however, are based only on work on paper and do not consider the influence of a display in PC work or similar work. A high illuminance environment has a problem such as causing it difficult to see the screen when working with a display screen. Since brightness preference differs by individuals, it is considered necessary to vary an illuminance environment by an individual's preference and the nature of work rather than to use a constant illuminance environment in the same indoor space.

\subsection{Chromaticity}

Chromaticity is a measure of color used for object color and light color. While there are multiple ways to represent chromaticity, this experiment uses RGB, XYZ, and $\mathrm{L} * \mathrm{a} * \mathrm{~b} *$ systems. The XYZ color system is a standard color coordinate system defined by CIE, and values of other color systems such as the XYZ or L*a*b* color system can be obtained by converting values of the XYZ color system. Since color recognition is based on human sensitivity, it is not possible to represent color only by the energy intensity of light. The XYZ color system, however, quantifies color, which is a sensible quantity, by numericizing the intensity of color sensation obtained by cones, color sensing organs, from the visible light spectrum. This numericized intensity of color sensation from the visible light spectrum is called a color matching function. A triple of values $(\mathrm{X}, \mathrm{Y}, \mathrm{Z})$ representing a color can be obtained from color matching functions and a spectrum distribution of light. XYZ chromaticity is calculated by Formulae 1, 2, and 3:

$$
\begin{aligned}
X & =\sum_{\lambda=380}^{780} I(\lambda) \bar{x}(\lambda) d \lambda \\
Y & =\sum_{\lambda=380}^{780} I(\lambda) \bar{y}(\lambda) d \lambda \\
Z & =\sum_{\lambda=380}^{780} I(\lambda) \bar{z}(\lambda) d \lambda
\end{aligned}
$$

$$
\begin{gathered}
\lambda \text { : wavelength }[\mathrm{nm}] I(\lambda): \text { spectrum intensity } \\
x^{-}(\lambda), y^{-}(\lambda), z^{-}(\lambda): \text { color matching functions }
\end{gathered}
$$

Of the XYZ chromaticity coordinates, Y represents brightness, and $X$ and $Z$ represent a change in the shade of color. It is difficult to grasp the relation between these $\mathrm{XYZ}$ values and color, and the amount of a change in those values is not in proportion to that in perception. For this reason, a degree of difference between different colors (color difference) cannot be quantitatively represented using those values. Therefore, the $\mathrm{L}^{*} \mathrm{a} * \mathrm{~b}$ color system is used when color difference needs to be represented. The $\mathrm{L}^{*} \mathrm{a}$ b $\mathrm{b}$ color system is a chromaticity system obtained by conversion from XYZ values. $\mathrm{L}^{*}$ value corresponds to brightness, $\mathrm{a}^{*}$, to a change in the shade of color from red to green, and $b^{*}$, such a change from yellow to blue. The $\mathrm{L}^{*} \mathrm{a} \mathrm{b}^{*}$ color system defines a standard color called white point. As $\left(a^{*}, b^{*}\right)=(0,0)$ is applied for the white point color, $\mathrm{L}^{*} \mathrm{a}^{*} \mathrm{~b}^{*}$ values obtained by measurement are relative to the white point. If a different color is used as the white point, the chromaticity coordinate of the same color changes. For this reason, the standard light source D65 is used as the white point of the $\mathrm{L} * \mathrm{a} * \mathrm{~b} *$ color system in this paper. The XYZ chromaticity coordinates are converted into the $\mathrm{L}^{*} \mathrm{a} * \mathrm{~b} *$ chromaticity coordinates by formulae 4,5 and 6 :

$$
\begin{aligned}
L^{*} & =116\left(Y / Y_{n}\right)^{\frac{1}{3}}-16 \\
a^{*} & =500\left[\left(X / X_{n}\right)^{\frac{1}{3}}-\left(Y / Y_{n}\right)^{\frac{1}{3}}\right] \\
b^{*} & =200\left[\left(Y / Y_{n}\right)^{\frac{1}{3}}-\left(Z / Z_{n}\right)^{\frac{1}{3}}\right]
\end{aligned}
$$

$$
X, Y, Z: \mathrm{XYZ} \text { values }
$$

$X_{n}, Y_{n}, Z_{n}: \mathrm{XYZ}$ values of the white point

Since the $\mathrm{L}^{*} \mathrm{a} * \mathrm{~b} *$ color system is so constituted that a change in color in the color space is approximately uniform, color difference can be represented by taking the Euclidean distance between two points in the color space.

The color difference $\Delta \mathrm{E}$ between Color 1 and Color 2 is calculated by Formula 7:

$$
\begin{gathered}
\Delta E=\sqrt{\left(L^{*}-L^{* \prime}\right)+\left(a^{*}-a^{* \prime}\right)+\left(b^{*}-b^{* \prime}\right)}(7) \\
L^{*}, \quad a^{*}, b^{*}: \text { color information of Color } 1 \\
L^{* \prime}, \quad a^{* \prime}, \quad b^{* \prime}: \text { color informaiton of Color } 2
\end{gathered}
$$


JIS specifies the tolerance for color difference in industrial use in stages. The color difference distinguishable by visual observation is specified to be 3.2 or greater, and two colors are recognized as different if the color difference between them exceeds 25 [8]. In this experiment, based on these values of color difference $\Delta \mathrm{E}$, it is judged whether the target chromaticity space is realized or not. As each of primary colors is emitted by a full color LED light source, the chromaticity of a full color LED light source can be represented as the intensity of primary colors relative to the visible light spectrum.

\subsection{Three Elements of Color}

There are three measures of color as its three elements: hue, luminosity, and saturation. Any color is located somewhere in the color solid constituted by these three elements.

Hue is a measure of a particular shade of color such as red, green, or blue. A circle in which hues are arranged is called a color wheel. Luminosity is a measure of darkness or lightness of a color. Greater its luminosity is, lighter a color is. When its luminosity reaches $100 \%$, a color becomes white. When its luminosity reaches $0 \%$, a color becomes black. Whereas hue indicates a particular shade of color such as red, blue, or green, colors with the same hue are recognized as different if they differ in luminosity. In the $\mathrm{L}^{*} \mathrm{a} * \mathrm{~b}^{*}$ color system used in this experiment, $\mathrm{L}^{*}$ represents luminosity.

Saturation is a measure of the vividness of a color. Greater its saturation is, more vivid a color is. A color whose saturation is 0 is called an achromatic color, and white, black, and different shades of gray fall under achromatic colors. Fig. 1 shows the relation among three elements of color.

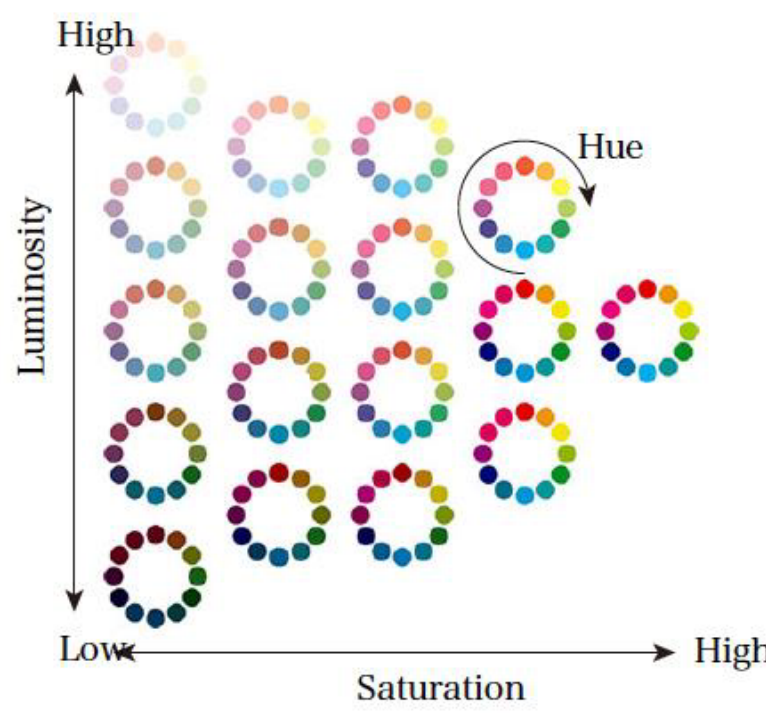

Figure 1. Three Elements of Color.

\subsection{Color mixing}

There are two ways of mixing colors: additive color mixing, a way of mixing light source colors, and subtractive color mixing, a way of mixing object colors. Additive color mixing results in a lighter color, while subtractive color mixing results in a darker color.

\section{Additive Color Mixing}

Additive color mixing refers to mixing of light source colors. Three colors of red, blue, and green are primary colors of light. If you mix these three colors, you get white. Fig. 2 shows three primary colors of light. In this way, mixing colors by additive color mixing results in a lighter color. Formulae 8, 9, and 10 for additive color mixing are given below:

$$
\begin{aligned}
& R= \begin{cases}255 & (\text { R1 } 22>255) \\
R 1+R 2 & \text { (otherwise })\end{cases} \\
& G= \begin{cases}255 & (\text { G1 } 1+\text { G } 2525) \\
G 1+G 2 & \text { (otherwise })\end{cases} \\
& B= \begin{cases}255 & (\text { B1 }+ \text { B } 2>255) \\
B 1+B 2 & \text { (otherwise })\end{cases}
\end{aligned}
$$

$R, G, B$ : color information of a color resulting from additive color mixing

$R 1, G 1, B 1$ : color information of Color 1 $R 2, G 2, B 2$ : color information of Color 2

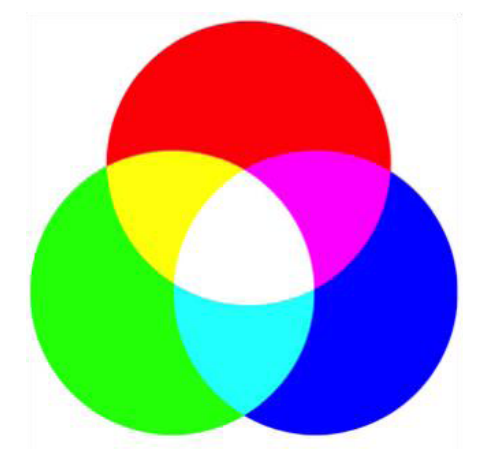

Figure 2. Three Primary Colors of Additive Color Mixing. Subtractive Color Mixing

Subtractive color mixing refers to mixing of object colors. Magenta, cyan, and yellow are three primary colors of pigment. If you mix these three colors, you get black. Fig. 3 shows three primary colors. In this way, mixing colors by subtractive color mixing results in a darker color. Formulae 11, 12, and 13 for subtractive color mixing are given below:

$$
\begin{aligned}
& R= \begin{cases}0 & ((255-R 1)+(255-R 2)>255) \\
255-((255-R 1)+(255-R 2)) & \text { (otherwise })\end{cases} \\
& G= \begin{cases}0 & ((255-G 1)+(255-G 2)>255) \\
255-((255-G 1)+(255-G 2)) & \text { (otherwise })\end{cases} \\
& B= \begin{cases}0 & ((255-B 1)+(255-B 2)>255) \\
255-((255-B 1)+(255-B 2)) & \text { (otherwise) }\end{cases}
\end{aligned}
$$

$R, G, B$ : color information of a color resulting from subtractive color mixing

$R 1, G 1, B 1$ : color information of Color 1 $R 2, G 2, B 2:$ color information of Color 2 


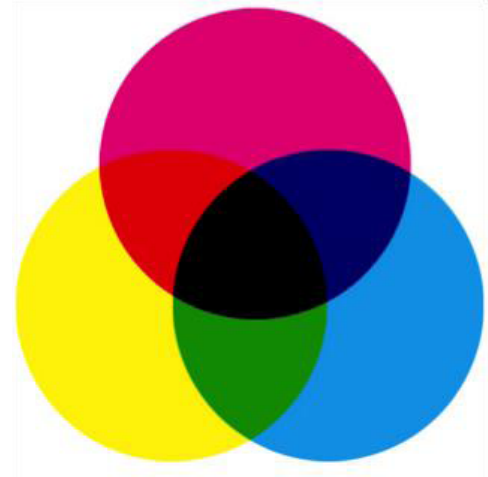

Figure 3. Three Primary Colors of Subtractive Color Mixing.

\section{Color-Lighting Control System using Colored Paper User Interface}

In this study, the Color-Lighting Control using Colored Paper User Interface (CLC/CPUI) is developed. The purpose of CLC/CPUI is to enable anyone to control full color LED lamp in an intuitive manner. A full color LED lamp is to be feedback-controlled by sensing a piece of colored paper as UI by a vision sensor to realize colored light demanded by a user. In CLC/CPUI, light color demanded by a user is determined by coloring a piece of paper serving as UI with a crayon or color pencil. In this way, CLC/CPUI has a feature that it is accessible even to a user unaccustomed to the operation of information device because of its UI using a piece of paper, a crayon, and a color pencil.

CLC/CPUI is implemented by connecting a vision sensor, a full color LED lamp, and a control computer to the same network. A piece of paper serving as UI is placed within the range of the vision sensor. The configuration diagram of CLC/CPUI is shown in Fig. 4.

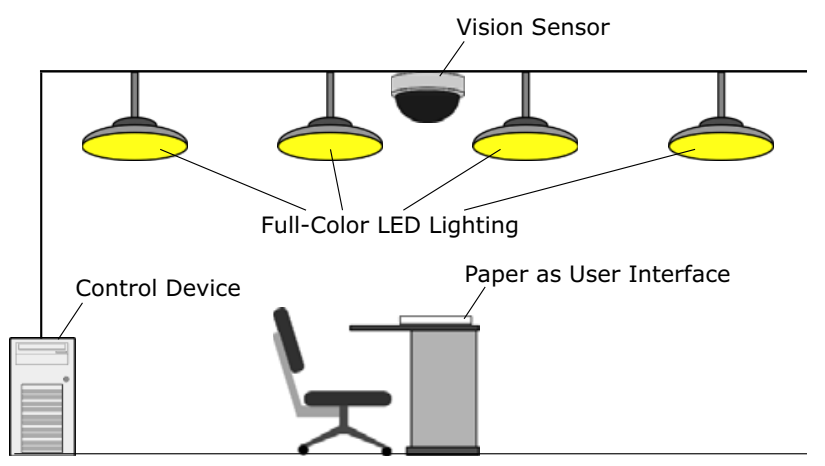

Figure 4. System Configuration of CLC/CPUI.

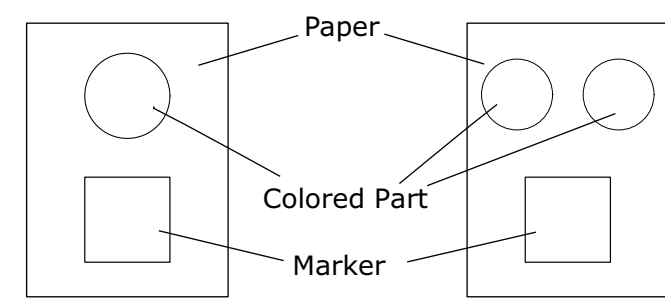

Monochromatic
Figure 5. Paper as User Interface.

\subsection{Paper UI}

CLC/CPUI uses a piece of paper as UI. There are two types of UI: one used in monochromatic light control and one used in mixed color light control. Fig. 5 shows paper UI used in monochromatic light control and mixed color light control.

\subsection{Method for Sensing UI in Monochromatic Light Control}

A piece of paper to be used as UI has areas to be colored and sensed. A user colors the area to be colored with a crayon in the color he/she wants. The current colored light environment can be detected by sensing the RGB values of the sensing area with a vision sensor.

Shown below is the flow of sensing the current colored light environment with a vision sensor from a piece of paper used as UI.

1. Detect the sensing area of a piece of UI paper.

2. Obtain the RGB values of the sensing area.

By performing the above operations, the RGB values of the colored light environment demanded by a user and that of the current environment can be compared.

\subsection{Method for Sensing UI in Mixed Color Light Control}

A piece of paper to be used as UI has two areas to be colored and a sensing area. A user colors those areas with a crayon in the colors he/she wants.

Shown below is the flow of determining the color demanded by a user on the basis of the color of two areas to be colored.

1. Detect two colored areas on a piece of UI paper.

2. Obtain the RGB values of each colored area.

3. Mix two RGB values by subtractive color mixing.

By performing the above operations, the color information of colored light demanded by a user can be obtained

The sensing area is sensed in the same way as it is sensed when there is a single UI circle to detect the current colored light environment, which is compared with the environment that the user wants.

\subsection{Controlling Algorithm of CLC/CPUI}

CLC/CPUI is feedback-controlled on the basis of values of color information extracted from images transmitted regularly to the control computer by the vision sensor which senses UI paper. By repeating this feedback control process, the target colored light environment is to be provided. Equation 14 gives the objective function used for searching the target colored light in CLC/CPUI. 


$$
f_{i}=\sqrt{\left(L^{*}-L^{* \prime}\right)+\left(a^{*}-a^{* \prime}\right)+\left(b^{*}-b^{* \prime}\right)}(14)
$$

$L^{*}, a^{*}, b^{*}:$ color information of the target colored light

$L^{* \prime}, a^{* \prime}, b^{* \prime}$ : color information of the current colored light

CLC/CPUI realizes the target colored light by controlling the objective function formalized as Equation 14 so as to minimize its value. The flow of CLC/CPUI control is shown below.

1. Turn on a LED lamp with the color set to white.

2. A user draws a circle on a piece of UI paper in any color to choose the target colored light.

3. Place the UI created in the range of the vision sensor.

4. The vision sensor obtains a UI image.

5. Analyze the image to obtain the RGB values of the color requested by the user.

6. Vary the luminance randomly in the neighborhood of $\pm 20 \%$.

7. The vision sensor obtains a UI image.

8. Analyze the image to obtain the RGB values of the current color environment.

9. Calculate the objective function on the basis of color information obtained in (8).

10. The vision sensor obtains a UI image.

11. Analyze the image to obtain the RGB values of the current color environment.

12. Calculate the objective function. If its value is better, then go to (6).

13. If the value of the objective function is worse, then revert the colored light to its previous state and go to (6).

The above operations constitute a single step of control. The element of LED lighting for each color is controlled. The colored light environment demanded by the user can be provided by repeated control.

\section{Evaluation of CLC/CPUI}

It is verified whether the target colored light environment is realized by using CLC/CPUI. In the verification experiment, an LED lamp is controlled by using CLC/CPUI toward the target colored light, and it is evaluated whether the target colored light is realized on the basis of chromaticity described earlier in Section 2.3.

\subsection{Verification of the Precision of CLC/CPUI for Monochromatic Light}

The verification experiment was conducted at the Intelligent Systems Design Laboratory, Kochikan Building, Doshisha University. The experiment was conducted by using 29 full color LED lamps controllable in four colors of red, blue, green and yellow made by SHARP, 1 iPhone $5 \mathrm{~s}$ as a vision sensor, 1 control computer, and pieces of paper on which a circle in an arbitrary color is drawn. A single circle was printed on pieces of paper as UI. There are three colors of the circle: Color 1, Color 2, and Color 3. RGB values of each color is as follows: [254, 160, 186] for Color $1,[31,80,77]$ for
Color 2, and [34, 38, 138] for Color 3. The target RGB values were the same as those for the painted color.

Fig. 6 gives the diagram of the experimental environment. Fig. 6 (a) is the plan of the experimental environment, and Fig. 6 (b) is the LED layout drawing. Fig. 7 shows a scene of the experiment.

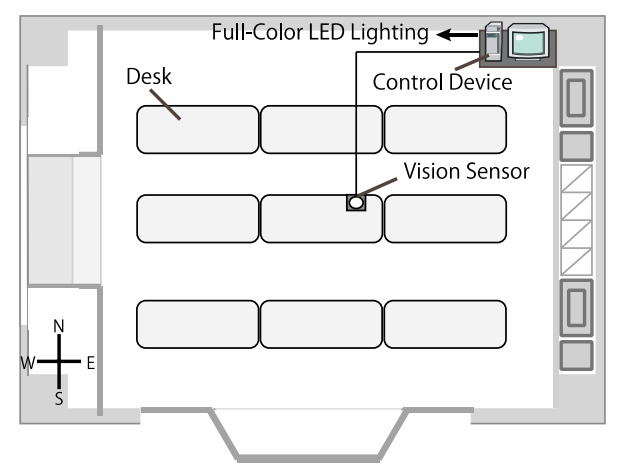

(a)Plan View

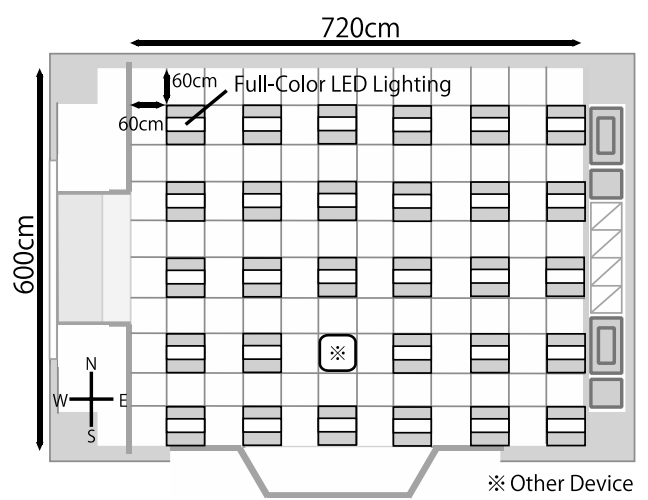

(b)Plot Plan of Full-Color LED Lighting

Figure 6. Experimental Environment

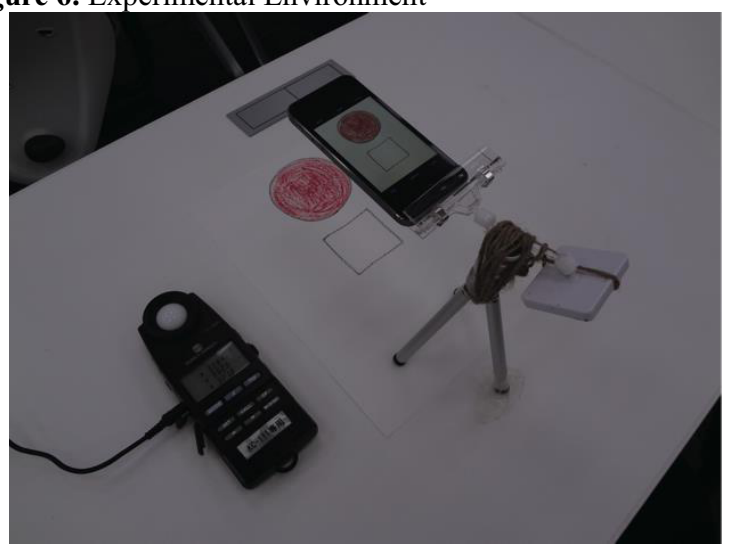

Figure 7. Experimental Landscape.

An experiment was conducted to verify that the target monochromatic light can be realized by using CLC/CPUI. There were three colors for a circle drawn on the piece of UI paper: Colors 1, 2, and 3.

The target colored light was set to have Color 1, 2, or 3. Fig. 8 shows the record of color difference under CLC/CPUI. In Fig. 8, the vertical axis represents the color difference between the target light environment and the current light environments, and the horizontal axis represents the number of control steps. The color 
difference after 300 steps was 21.7 with Color $1,21.9$ with Color 2, and 2.2 with Color 3.

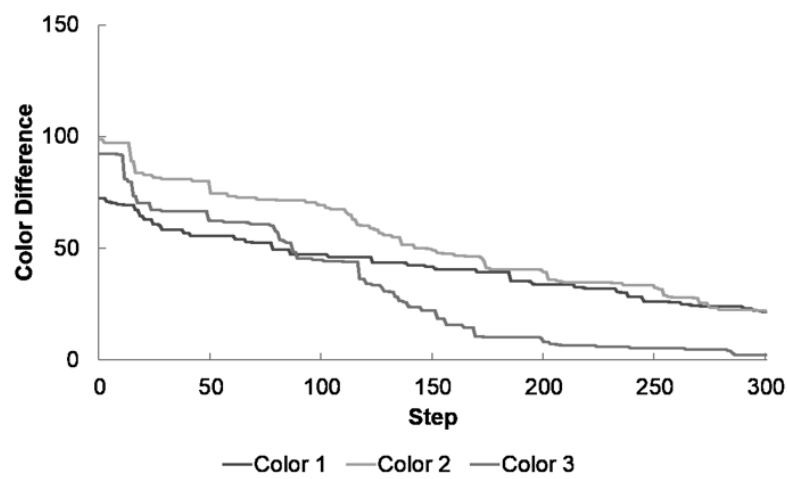

Figure 8. Result of CLC/CPUI for monochromatic light.

\subsection{Verification of the Precision of CLC/CPUI for Mixed-Colored Light}

The verification experiment was conducted at the Intelligent Systems Design Laboratory, Kochikan Building, Doshisha University. The experiment was conducted by using 29 full color LED lamps made by SHARP whose light sources in four colors of red, blue, green and yellow were controllable, 1 iPhone $5 \mathrm{~s}$ as a vision sensor, 1 control computer, and pieces of paper on which a circle in an arbitrary color is drawn. Two circles were drawn on a single piece of UI paper with three input colors: Colors 4, 5, and 6. RGB values for Color 4 were [225, 58, 249], those for Color 5, [255, 250, 53], and those for Color 6, [93, 250, 253]. Two out these three colors are chosen to color circles on the piece of UI paper. Pieces of UI paper were created in three patterns. The result of mixing Colors 4 and 5 was named Pattern A, that of mixing Colors 5 and 6 , Pattern B, and that of mixing Colors 4 and 6, Pattern C. Fig. 9 shows Patterns $\mathrm{A}, \mathrm{B}$, and $\mathrm{C}$.
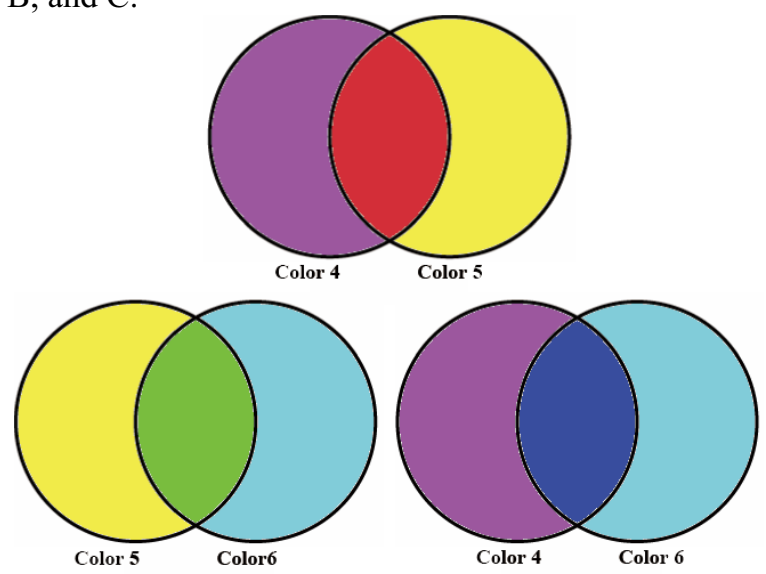

Figure 9. Mixed Color in three Patterns.

An experiment was conducted to verify that the target mixed-colored light can be realized by using CLC/CPUI. Fig. 10 shows the record of color difference when CLC/CPUI was controlled for the target colored light determined by three UI patterns. In Fig. 10, the vertical axis represents the color difference between the target light environment and the current light environment, and the horizontal axis represents the number of control steps.
The color difference after 300 steps was 27.5 with Pattern A, 43.8 with Pattern B, and 0.3 with Pattern C.

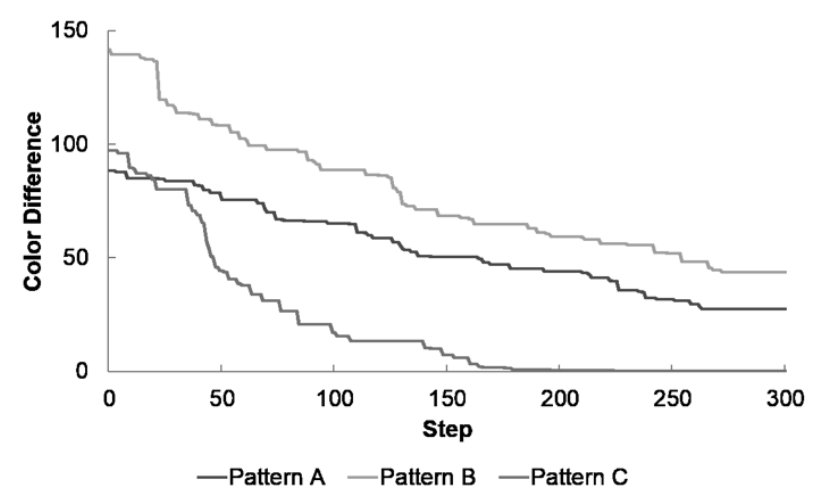

Figure 10. Result of CLC/CPUI for Mixed-Colored Light.

\subsection{Verification of the Precision of CLC/CPUI for Mixed-Colored Light with Luminosity Fixed}

This verification experiment was conducted in a dark room where any lamps other than the controlled lamp were turned off. The experiment was conducted by using 1 LED lamp Hue, made by Phillips, whose light sources in three colors of red, blue, and green were controllable, 1 iPhone $5 \mathrm{~s}$ as a vision sensor, 1 control computer, and pieces of paper on which a circle in an arbitrary color is drawn. Two circles were drawn on the piece of UI paper, similarly, with three input colors: Colors 4,5 , and 6 . RGB values for Color 4 were $[225,58,249]$, those for Color 5, $[255,250,53]$, and those for Color 6, [93, 250, 253]. Two out these three colors are chosen to color circles on a single piece of UI paper. Pieces of UI paper were created in three patterns. The result of mixing Colors 4 and 5 was named Pattern A, that of mixing Colors 5 and 6, Pattern B, and that of mixing Colors 4 and 6, Pattern C. Fig. 9 shows Patterns A, B, and C.

An experiment was conducted to verify that the target mixed-colored light can be realized by using CLC/CPUI when luminosity was fixed. Fig. 11 shows the record of color difference when CLC/CPUI was controlled for the target colored light determined by three UI patterns. In Fig. 11, the vertical axis represents the color difference between the target light environment and the current light environment, and the horizontal axis represents the number of control steps. The color difference after 300 steps was 21.9 with Pattern A, 28.7 with Pattern B, and 6.8 with Pattern C.

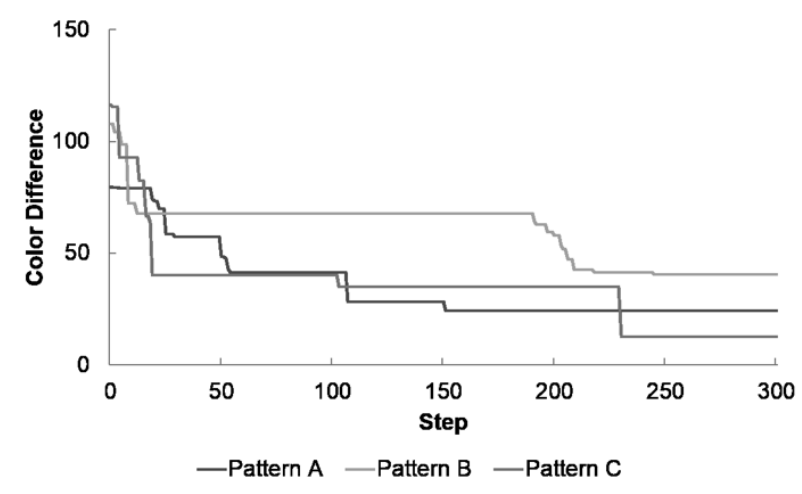


Figure 11. Result of CLC/CPUI for Mixed-Colored Light with Luminosity Fixed.

\subsection{Discussion}

It was found that, if a lamp is controlled by using CLC/CPUI for arbitrary colored light, colored light from the lamp approaches the target, both with monochromatic input color and mixed input color. According to the tolerance for color difference in industrial use prescribed in JIS, two colors are to be recognized as different if color difference between them exceeds 25.0. With the above result, when a single color was put into UI, color difference was 25.0 or less, whichever of piece of papers on which a circle was drawn in Color 1, 2, or 3 was used as UI. Therefore, the optimal solution is said to have been found in colored light environment search. On the other hand, when two colors were put into UI, there were cases where color difference was not 25.0 or less. The optimal solution failed to be found in colored light environment search. In this way, it is considered that there are both cases where the colored light environment demanded can be provided on the basis of color information of the target colored light and those where it cannot be provided. It is possible that this problem was caused by the inability to find the optimal solution due to a change in luminosity that was simultaneous with a change in the chromaticity of colored light.

CLC/CPUI is thus modified so that the luminosity of colored light does not change and then evaluated. As a result, it was found that controlling a lamp to produce arbitrary colored light by using modified CLC/CPUI which did not cause a change in luminosity resulted in colored light closer to the target. Even in this case, however, there was a case where color difference is not 25.0 or less, failing to find the optimal solution. Since lessening the number of parameters resulted in colored light closer to the target, a more efficient search for the optimal solution is considered to be possible by changing conditions.

\section{Conclusion}

This study, in order to realize an intuitive colored light control system, constructed a Color-Lighting Control System using Colored Paper User Inter-face (CLC/CPUI) and conducted verification experiments. CLC/CPUI attempts to bring colored light produced by a lamp closer to one demanded by a user through the feedback control using a vision sensor. Verification experiments for CLC/CPUI were conducted in three cases: for monochromatic light, mixed color light, and mixed color light with luminosity fixed. It was found that colored light from a lamp can be brought closer to the target by using CLC/CPUI in any of those cases.

It was found that, if a monochromatic color is input, colored light approached the target and converged within the tolerance. On the other hand, if a mixed color is input, colored light approached the target but sometimes failed to converge within the tolerance. It is possible that this problem was caused by the inability to find the optimal solution due to a change in luminosity that was simultaneous with a change in the chromaticity of colored light.

CLC/CPUI is thus modified so that the luminosity of colored light does not change simultaneously when its chromaticity is changed and then evaluated. Even in this case, colored light sometimes failed to fall in the tolerance. It became easier, however, to find the optimal solution and colored light was brought closer to the tolerance as luminosity was fixed and parameters were reduced to chromaticity only.

\section{References}

1. F. Obayashi, K. Tomita, Y. Hattori, etc. "A Study on Environmental Control Method to Improve Productivity of Office Workers -Development of an Illumination Control Method and its Experimental Evaluation," Human Interface Symposium 2006, No.1322, pp.151-156 (2006).

2. M. Miki, Y. Taniguchi, M. Yoshimi, "Effect of Preferred Illuminance and Color Temperature on Creative Works/Intellectual Productivity," Journal of the Illuminating Engineering Institute of Japan, 96[8], 437-441 (2012)

3. PHILIPS. ActiViva club.

4. Dong gi Lee, Hsin chen Chiang, Takaaki Koga, Kotaroh Hirate, "Basic Study on Discomfort Caused by LED Lighting Colors in Working Space," AIJ Journal of Technology and Design, Vol. 17, No.35, pp.201-204 (2011).

5. A. Chand, A. K. Dey "Jadoo: A Paper User Interface for Users Unfamiliar With Computers," Proc. CHI2016, pp.1625-1630 (2006).

6. P.R.Boyce, N.H.Eklund and S.N.Simpson, "Individual Lighting Control: Task Performance, Mood, and Illuminance", Journal of the Illuminating Engineering Society, 131-142 (2000).

7. JIS. JISZ9110. 2010

8. JIS. JISZ8721.1993. 